\section{Anerkennung in erweiterter Arbeit}

\author{
Ergebnisse der Dissertation
}

von Linda Nierling, ITAS

Wie kann der Krise der Arbeitsgesellschaft begegnet werden? In den Debatten der 1980er und 1990er Jahre wurden alternative Arbeitsformen als gesellschaftliches Lösungsmodell intensiv diskutiert. Allerdings gibt es bis heute keine breite Umsetzung dieser Ideen. In der hier vorgestellten Dissertation wird an diese Lücke durch eine Analyse von Anerkennungsverhältnissen bezahlter und unbezahlter Arbeit angesetzt.

Die Diagnose der „Krise der Erwerbsarbeit“ wird seit 30 Jahren immer wieder zur Beschreibung gegenwärtiger Arbeitsverhältnisse herangezogen. Allerdings hat trotz aller ,krisenhaften“ Befunde Erwerbsarbeit ihre zentrale Rolle in der Gesellschaft bislang nicht verloren. Vielmehr bleibt bezahlte Arbeit Gradmesser für gesellschaftliche Teilhabe und wirkt bestimmend für die Ausbildung der personalen Identität. Von dieser gegenläufigen Entwicklung ausgehend, knüpft die Dissertation (vgl. Nierling 2013) an Debatten an, in denen die „Krise der Arbeitsgesellschaft" vor dem Hintergrund schwindender Industriearbeit verhandelt wurde. Dabei wurde Erwerbsarbeit in ihrer Bedeutung als zentraler gesellschaftlicher Integrationsmodus grundsätzlich in Frage gestellt. Bei der Suche nach Alternativen kamen unbezahlte Arbeitsformen wie Familienarbeit, Ehrenamt und Eigenarbeiten als bisher unbeachtete Ressourcen für ein „neues“ Verständnis von Arbeit in den Blick, wodurch die hohe individuelle und gesellschaftliche Bedeutung der Erwerbsarbeit sowohl ideell als auch zeitlich zugunsten von Nicht-Erwerbsarbeit relativiert werden sollte. Blickt man aus heutiger Perspektive auf diese Debatten zurück, so wird einerseits deutlich, dass der Sprung zur gesellschaftlichen Umsetzung dieser theoretischen Konzepte - politisch - nicht erfolgt ist. Auch fehlt - in wissenschaftlicher Hinsicht - bislang eine konkrete Auseinandersetzung damit, welche subjektive Bedeutung nicht-erwerbsförmige im Vergleich zu erwerbsförmigen Tätigkeiten haben. An dieser Lücke der wissenschaftlichen Auseinandersetzung setzt die
Dissertation an, die für diese Untersuchung „Anerkennung" in der Konzeption von Axel Honneth (1994) als Analysekategorie verwendet.

\section{Erweiterte Konzepte von Arbeit in den 1980 er und 1990er Jahren}

Hervorgerufen durch die Wirtschaftskrise der 1970er Jahre sowie umfassende Automatisierungs- und Effizienzstrategien in der Industrie fielen viele industrielle Arbeitsplätze weg, und die zuvor stabilen Arbeitsmarktbedingungen Nachkriegsdeutschlands erodierten. Die politischen Debatten waren in Deutschland in diesen Jahren stark von der Diagnose der „Massenarbeitslosigkeit" geprägt. In den 1990er Jahren kamen diskontinuierliche Arbeitsbiografien (,Erosion des Normalarbeitsverhältnisses") auch in hochqualifizierten Berufsfeldern hinzu. Für Erwerbstätige verursachte der wachsende Druck globaler Märkte, dem Staat und Unternehmen durch Deregulations- und Flexibilisierungsstrategien begegneten, eine steigende Erwerbsunsicherheit (Kocka/Offe 2000; Schmid 2000). Diese Entwicklung hält durch prekäre Arbeitsbedingungen und eine hohe Sockelarbeitslosigkeit bis heute an (Hradil 2005). Neben politischen Strategien einer Deregulierung der Arbeitsmärkte oder einer Umverteilung von Arbeit durch Arbeitszeitverkürzungen wurde auch das Erwerbsarbeitsmodell an sich auf den Prüfstand gestellt (Senghaas-Knobloch 2000). Dieser dritte Vorschlag, der später auch in der Debatte um Nachhaltigkeit aufgegriffen wurde, sah einen neuen gesellschaftlichen Arbeitsvertrag vor, in dem bezahlte und unbezahlte Arbeit als zentrale Komponenten miteinander kombiniert wurden (Kambartel 1994). Hierfür war die Annahme zentral, dass bezahlte Arbeit das Arbeitsvermögen einer Gesellschaft nur unzureichend abbildet. Vielmehr gibt es eine Vielzahl von Arbeiten, die insbesondere von Frauen geleistet werden, die nicht als „Arbeit“" anerkannt werden, wie Familienarbeit, Freiwilligenarbeit oder auch Eigenarbeit (Bock/Duden 1977; Kambartel 1994; Kontos/Walser 1978). Eine Weitung des gesellschaftlichen Verständnisses von Arbeit fand statt (vgl. u. a. Biesecker 2000; Peinl 2003).

In Folge der Debatten wurden vielen Modelle und Konzepte entwickelt, die die praktische Überführung solcher Ideen von gesellschaftli- 
cher Arbeit zum Ziel hatten, z. B. die „Multiaktivitätsgesellschaft“ (Gorz 2000), die „Neue Arbeit" (Bergmann 2004), das „Ganze der Arbeit" (Biesecker 2000) oder die „Bürgerarbeit“ (Beck 1999). Den meisten Ansätzen ist gemein, dass sie Entkopplung von Existenzsicherung und bezahlter Arbeit befürworten, sowie eine Neuverteilung von Zeit fordern, um Räume für unbezahlte Tätigkeiten zu schaffen. Gleichzeitig geht es auch darum, ein neues Verständnis von Lebensqualität zu schaffen, das im Sinne postmaterieller Wertvorstellung den steten Wachstums- und Konsumparadigmen der Industriegesellschaft Werte wie eine aktive Zivilgesellschaft, Stärkung des gesellschaftlichen Zusammenhalts durch die Integration von Erwerbslosen, der Stabilisierung zwischenmenschlicher Beziehungen durch gegenseitige Zuwendung oder die Aktivierung von eigenem kreativem Potenzial gegenüberstellt.

Die Debatten um einen weiten Arbeitsbegriff und der Öffnung des Arbeitsmarktes erscheinen auf der normativen und theoretischen Ebene unmittelbar einleuchtend, da nur durch sie nachhaltige Konsummuster oder auch Formen der politischen Partizipation gestärkt werden können. Allerdings haben sie sich bislang noch nicht auf breiter gesellschaftlicher Ebene durchgesetzt. Auch gibt es bislang nur wenige empirische Untersuchungen, die in den Blick nehmen, warum sich die Konzepte nicht auf eine praktische Ebene überführen lassen. An diese Lücke setzt die Dissertation mit einer qualitativen Fallstudienuntersuchung zu verschiedenen Formen erweiterter Arbeit an.

\section{Analysekategorie Anerkennung}

Ausgehend von der Fragestellung, welchen Beitrag erweiterte Arbeitskonzepte innerhalb des gesellschaftlichen Arbeitsvertrages leisten können, wird „Anerkennung“ in der Konzeption von Axel Honneth (1994) als Interpretationsrahmen herangezogen. Die Perspektive der Anerkennung ermöglicht es, subjektive Arbeitserfahrungen und -motive auf individueller Ebene zu analysieren. Hilfreich ist die Systematisierung von „Anerkennung" durch Honneth in die drei Dimensionen „Recht", „Solidarität" und „Liebe“ (vgl. Honneth 1994; Honneth 2004; Iser 2008). Unter „Recht“ versteht Honneth die gegenseitige Anerkennung individueller Rechte durch alle Mitglieder der Gesellschaft, die den Subjekten Selbstachtung ermöglicht. Die Anerkennungsform „Recht“ wird von den Individuen in sozialstaatlichen Rechten und Pflichten erfahren, die über staatliche Institutionen oder Mechanismen in Organisationen vermittelt werden. „Solidarität" umfasst demgegenüber die Anerkennung bestimmter Beiträge von Individuen zu gesellschaftlichen Zielen, auf deren Grundlage eine Selbsteinschätzung eigener Befähigungen und Leistungen realisiert werden kann. Unter ,Solidarität“ können alle Formen und Zeichen der Bestätigung gefasst werden, wodurch Individuen ihre Fähigkeiten und Kompetenzen in gesellschaftlichen Werthorizonten anerkannt bzw. missachtet sehen. Anerkennung im Modus „Liebe" findet sich in nahen emotionalen Beziehungen und bestärkt die Individuen in ihren Gefühlen und Bedürfnissen. Sie umfasst alle Formen der Interaktion, in denen sich Individuen in ihrer Persönlichkeit von anderen Personen positiv bestätigt oder durch sie missachtet fühlen. Folglich lassen sich intersubjektive Anerkennungsverhältnisse durch persönliche (Liebe), leistungsbezogene (Solidarität) und institutionalisierte (Recht) Formen der Anerkennung fassen. Diese Systematisierung lässt sich in grundsätzlicher Weise auch auf das Feld erweiterter Arbeit anwenden. Sie bedarf jedoch der theoretischen Ausdifferenzierung, um den Besonderheiten erweiterter Arbeit Rechnung zu tragen, die an anderer Stelle ausführlich vorgenommen wurde (vgl. Nierling 2013).

\section{Empirische Ergebnisse}

Erfahrungen von Anerkennung und Missachtung sind eng mit biografischen Prozessen und subjektiven Alltagserfahrungen verknüpft. Um Anerkennungschancen, die ein Konzept erweiterter Arbeit haben kann, zu erheben, wurde ein qualitatives Forschungsdesign gewählt (Flick et al. 2007). Als methodisches Untersuchungsdesign wurde hierbei die Forschungsstrategie Fallstudie gewählt (z. B. Yin 2003). Es wurden drei Fallstudien erhoben, Zentrum für Kreativität, Zukunft e.V. und Natur AG (Namen sind pseudonymisiert). Um ein möglichst umfassendes Bild von den Fällen zu erhalten, wurden problemzentrierte Interviews mit Leitungspersonen und MitarbeiterInnen der 
Organisationen geführt (Witzel 2000). So konnten zum einen Rahmenbedingungen, Aufgaben, Anforderungen von erweiterter Arbeit in diesem Fall erfasst werden, zum anderen war es möglich, subjektive Arbeitserfahrungen $\mathrm{zu}$ erheben. Insgesamt wurden 27 Interviews geführt. Die Interviews fanden in den Jahren 2008 und 2009 statt. Die Interviews wurden softwaregestützt nach den Regeln der qualitativen Inhaltsanalyse analysiert.

Im Zentrum für Kreativität, einer Non-ProfitOrganisation in einer deutschen Großstadt, können Menschen in kostenpflichtigen Werkstätten Produkte für den Eigenbedarf herstellen. Sie machen in diesen Arbeitsprozessen ergänzende Anerkennungserfahrungen zu denen der Erwerbsarbeit, die insbesondere in Phasen des biografischen Wandels eine besondere Bedeutung erfahren und oftmals Missachtungserfahrungen aus anderen Lebensumständen kompensieren können. Während rechtliche Anerkennungsstrukturen im Zentrum für Kreativität nicht stark ausgeprägt sind, finden entsprechende Anerkennungserfahrungen hier durch die informale Einbindung in die Organisation statt. Hinsichtlich individueller Anerkennungserfahrungen im Modus Solidarität entsteht durch die handwerkliche Arbeit ein positiver Bezug auf die eigenen Talente, Kompetenzen und Fähigkeiten, die oftmals im Zentrum für Kreativität reaktualisiert oder neu entdeckt werden. Die Menschen erfahren dadurch eine Bestätigung des subjektiven Leistungsvermögens, das insbesondere durch den persönlichen „Mehrwert", den die Produkte durch den individuellen Herstellungsprozess erhalten, unterstützt wird. Im Anerkennungsmodus Liebe erfahren die Menschen ebenfalls Bestätigung im Zentrum für Kreativität, da durch die gemeinsamen Schaffensprozesse Gefühle der Zugehörigkeit zu einer Gemeinschaft entstehen. Auch hier sind es die materiellen Arbeitsergebnisse, die die zwischenmenschliche Bestätigung und Entwicklungen von Beziehungen durch gegenseitiges Lob vermitteln, aber auch stärken.

Im Fall Zukunft e.V., einem Verein in einer strukturschwachen Region mit einer hohen Arbeitslosenquote, der sich zum Ziel gesetzt hat, arbeitslose Jugendliche an unbezahlte Arbeitsformen heranzuführen, sind die Anerkennungspotenziale erweiterter Arbeit nicht so eindeutig verteilt. Während sich ein Modell erweiterter Arbeit, d. h.
Mischformen von bezahlter und ehrenamtlicher Arbeit, für diejenigen, die den Verein gegründet haben, realisiert, bleibt für die übrigen Mitarbeiter, ebenso wie für die Jugendlichen bezahlte Arbeit das Referenzmodell. Dadurch kommt dem Anerkennungsmodus Recht und damit der formalen Einbindung im Verein eine hohe Bedeutung zu. So bewerten die Mitarbeiter, aber auch die Jugendlichen, ihren persönlichen Status im Verein dadurch, ob sie durch einen Arbeitsvertrag in die Organisation eingebunden sind oder ob sie derzeit arbeitslos sind und daher in keinem „regulären“ Verhältnis zum Verein stehen. Die Anerkennung im Modus Solidarität differiert ebenso zwischen den verschiedenen Gruppen im Verein. Während die Gründer des Vereins eine hohe externe Sichtbarkeit der eigenen Leistungen erfahren und Anerkennung für die durch ihre Kompetenzen umgesetzte Kreativität erfahren, fehlen den Mitarbeitern oftmals organisationale Referenzkriterien, durch die sie den Wert der eigenen Arbeitsleistung bestimmen können. Zudem stehen ihnen nur wenige Räume zur Verfügung, in denen sie eigene Ideen verwirklichen können. Auch die Jugendlichen, die dort einem „1-Euro-Job“ nachgehen, widmen sich fast ausschließlich fest vorgegeben Arbeitsaufgaben und haben selten die Gelegenheit, eigene Fähigkeiten und Kompetenzen selbstbestimmt zu erschließen. Schließlich überwiegen im Anerkennungsmodus Liebe enge und konflikthafte Bindungen, die oftmals mit Gefühlen der Ausgrenzung vom Verein bei Mitarbeitern und Jugendlichen verbunden sind.

In der Natur AG, ein an Prinzipien der Nachhaltigkeit orientiertes Unternehmen, das in einem umfassenden Work-Life-Balance-Programm versucht, seitens des Unternehmens eine gelingende Verbindung von Arbeit und Leben herzustellen, zeigt sich eine hohe individuelle Relevanz von Erwerbsarbeit bei den Mitarbeitern. Doch zeigt sich gerade im Gegensatz zu vergleichbaren Unternehmen (vgl. Hochschild 2006), dass die Mitarbeiter trotz einer hohen normativen Orientierung an der Erwerbsarbeit eine 40-Stunden-Woche realisieren, die für sie eine gute Grundlage für die Balance von Arbeit und Leben darstellt. Hinsichtlich der Erfahrung von Anerkennung hat der Anerkennungsmodus Recht für die Mitarbeiter eine hohe Bedeutung, nicht zuletzt, da sie in das Unternehmen mit allen 
damit verbundenen Rechten und Pflichten formal über einen Arbeitsvertrag eingebunden sind. Aber auch im Modus Solidarität erfahren die Mitarbeiter Anerkennung, was sich insbesondere in vorhandenen Möglichkeiten der persönlichen Weiterentwicklung niederschlägt. Dabei kommt dem Anerkennungsmodus Liebe in diesem Fall eine untergeordnete Bedeutung zu. So entstehen zwar persönliche Beziehungen innerhalb der Organisation, doch verbleiben diese in einer professionellen Distanz. Es zeigt sich allerdings, insbesondere bei Konflikten, dass die organisationalen Strukturen des Unternehmens auf emotionaler Ebene für die Mitarbeiter entlastend wirken.

In der vergleichenden Perspektive auf die drei Fälle zeigt sich zum ersten im Anerkennungsmodus Recht, dass die rechtlichen Anerkennungsunterschiede zwischen den unterschiedlichen Arbeitsformen so bestimmend sind, dass sich diese nicht ausgleichen lassen. Vielmehr setzen sich rechtlich begründete Prinzipien, die aus der Erwerbsarbeit hervorgehen, als Strukturen der Anerkennung und Missachtung in den Organisationen fort. Eine Kompensation dieser Erfahrungen ist über informale Regelungen dabei nur bedingt möglich.

Hinsichtlich des Modus Solidarität zeigt sich zweitens, dass in erweiterter Arbeit, in ganz unterschiedlicher Weise möglich, die eigene Leistung Bestätigung finden kann, sowohl durch Fremd- als auch durch Selbstbestätigung. In der Bestätigung durch andere sind für die Individuen Anerkennungsforen zentral, die ihnen die intersubjektive Sichtbarkeit von Leistung vermitteln, z. B. durch einen geteilten Orientierungsrahmen für den „Wert“ einer Leistung. Eine Selbstbestätigung des eigenen Leistungsvermögens findet sich insbesondere dann, wenn Individuen die Möglichkeit zur Selbstverwirklichung haben.

Im Anerkennungsmodus Liebe zeigt sich in vergleichender Perspektive drittens, dass unterschiedliche Tiefengrade der Beziehung für verschiedene Qualitäten der emotionalen Anerkennung verantwortlich sind, die gleichfalls Anerkennung und Missachtung vermitteln können. Zudem wird deutlich, dass gerade feste organisationale Regeln und Strukturen eine entlastende Wirkung haben und Individuen die Möglichkeit gewähren können, sich aus erlebter Missachtung zu befreien.

\section{Fazit: Individuelle Anerkennungschancen in erweiterter Arbeit}

Erweiterte Arbeitsformen bergen eine breite Vielfalt von „weichen“ Anerkennungserfahrungen, die sich auf individueller Ebene in gesellschaftlichen Nischen konstituieren können, so das Fazit der Untersuchung. Auf der Grundlage einer materiell gesicherten Basis können erweiterte Arbeitskontexte bei individueller Passung einen besonderen Wert für die Menschen einnehmen.

Zentral hierbei ist, dass sich in erweiterten Arbeitskontexten die Anerkennung von Leistung auch jenseits wirtschaftlicher Prinzipien realisiert: So stellen sich eigensinnige Zieldefinitionen und kontextabhängige Bezüge zur eigenen „Leistung“ ein, wodurch die intersubjektive Bewertung von Leistung auch jenseits ökonomischer Vermittlung, d. h. Geld, möglich wird. Zudem gestalten sich individuelle Anerkennungschancen in erweiterter Arbeit integrativ, da sie die ,ganze Person“ umfassen: Auf zwischenmenschlicher Ebene (Liebe) spielen Fähigkeiten und Kompetenzen (Solidarität) eine wichtige Rolle zur Beziehungsentwicklung und umgekehrt, durch die persönliche Bindung (Liebe) bekommt die Bestätigung der eigenen Fähigkeiten und Kompetenzen einen besonderen Wert. Auch bietet erweiterte Arbeit Raum für Selbstverwirklichung und für individuelle Suchprozesse. Dabei schaffen gerade diese autonom bestimmten Räume für das „Eigene“ dem Subjekt eine innere Befriedigung und Stärkung.

Warum hat sich ein Konzept erweiterter Arbeit bislang auf gesamtgesellschaftlicher Ebene nicht durchgesetzt? Zum einen sind (wirtschaftliche) Alternativen, z. B. Selbstversorgung, zur Erwerbsarbeit weitestgehend weggefallen, so dass die Strukturen des Arbeitsmarktes mehr und mehr bestimmend werden, wenn es um die Erlangung von Anerkennung und Missachtung in der Gesellschaft geht, was sich insbesondere in den Modi Recht und Solidarität widerspiegelt. Zum anderen lassen sich Missachtungserfahrungen, die z. B. aus der Arbeitslosigkeit entstehen, nicht durch unbezahlte Arbeitsformen kompensieren. So bilden materielle Sicherheit und förderliche Arbeitszeitregime die Grundlage für erweiterte Arbeit. Darauf aufbauend, auf der Basis von Freiwilligkeit, können jedoch subjektive Anerkennungschancen kontextabhängig entstehen. 
Welche Potenziale kann ein Konzept erweiterter Arbeit dennoch bieten? Kritisch ließe sich die Frage stellen, ob erweiterte Arbeit nur als Wohlstandskonzept für diejenigen funktioniert, die auf sicherer materieller Basis stehen und erweiterten Arbeitsformen neben ihrer geregelten Beschäftigung nachgehen. Diese Frage ist nicht zu verneinen, doch birgt erweiterte Arbeit gerade für diese Menschen große Potenziale. Sie haben die Möglichkeit, sich in erweiterten Arbeitsfeldern spezifische Anerkennungschancen in den Feldern Solidarität und Liebe zu erschließen, die an ihren subjektiven Bedürfnissen orientiert sind, sinnstiftend wirken und die eigene Lebensqualität erhöhen können. Auch können sie Missachtungserfahrungen aus anderen Lebensbereichen bis zu einem gewissen Grade kompensieren. Dies vermag Menschen im Sinne eines „Empowerments" dazu befähigen, Potenziale innerhalb ihrer eigenen Arbeits- und Lebensbezüge in emanzipatorischer Weise zu aktivieren, sich von restriktiven Strukturen des Arbeitsmarktes frei zu machen. Gerade in Zeiten einer fordernden Arbeitswelt birgt erweiterte Arbeit somit vielfältige Potenziale, die Individuen durch das Aufzeigen von Alternativen zum Bestehenden zu stärken.

\section{Literatur}

Beck, U., 1999: Modell Bürgerarbeit. In: Beck, U. (Hg.): Schöne neue Arbeitswelt. Vision: Weltbürgergesellschaft. Frankfurt a. M., S. 7-189

Bergmann, F., 2004: Neue Arbeit, neue Kultur. Freiamt Biesecker, A., 2000: Kooperative Vielfalt und das „Ganze der Arbeit“. Überlegungen zu einem erweiterten Arbeitsbegriff. Wissenschaftszentrum Berlin

Bock, G.; Duden, B., 1977: Arbeit aus Liebe - Liebe als Arbeit: Zur Entstehung der Hausarbeit im Kapitalismus. In: Frauen und Wissenschaft. Beiträge zur Berliner Sommeruniversität für Frauen, Juli 1976, S. 118-199

Flick, U.; Kardoff, E. v.; Steinke, I., 2007: Was ist qualitative Forschung? Einleitung und Überblick. In: Flick, U.; Kardoff, E. v.; Steinke, I. (Hg.): Qualitative Forschung. Ein Handbuch. Reinbek, S. 13-29

Gorz, A., 2000: Arbeit zwischen Misere und Utopie. Frankfurt a. M.

Hochschild, A.R., 2006: Keine Zeit. Wenn die Firma zum Zuhause wird und zu Hause die Arbeit wartet. Wiesbaden

Honneth, A., 1994: Kampf um Anerkennung. Zur moralischen Grammatik sozialer Konflikte. Frankfurt a. M.
Honneth, A., 2004: Recognition and Justice. Outline of a Plural Theory of Justice. In: Acta Sociologica 47/4 (2004), S. 351-364

Hradil, S., 2005: Soziale Ungleichheit in Deutschland. Wiesbaden

Iser, M., 2008: Empörung und Fortschritt. Grundlagen einer kritischen Theorie der Gesellschaft. Frankfurt a. M.

Kambartel, F., 1994: Arbeit und Praxis. In: Honneth, A. (Hg.): Pathologien des Sozialen. Die Aufgaben der Sozialphilosophie. Frankfurt a. M., S. 123-139

Kocka, J.; Offe, C. (Hg.), 2000: Geschichte und Zukunft der Arbeit. Frankfurt a. M.

Kontos, S.; Walser, K., 1978: Hausarbeit ist doch keine Wissenschaft! In: Beiträge zur feministischen Theorie und Praxis 1/1 (1978), S. 66-80

Nierling, L., 2013: Anerkennung in erweiterter Arbeit. Eine Antwort auf die Krise der Erwerbsarbeit? Berlin

Peinl, I., 2003: Geschlechterregime in der Erwerbsarbeit - Konturen der Gegenwart. In: Kuhlmann, E.; Betzelt, S. (Hg.): Geschlechterverhältnisse im Dienstleistungssektor. Dynamiken, Differenzierungen und neue Horizonte. Baden-Baden, S. 23-35

Schmid, G., 2000: Arbeitsplätze der Zukunft: Von standardisierten zu variablen Arbeitsverhältnissen. In: Kocka, J.; Offe, C. (Hg.): Geschichte und Zukunft der Arbeit. Frankfurt a. M., S. 269-292

Senghaas-Knobloch, E., 2000: Von der Arbeits- zur Tätigkeitsgesellschaft? Dimensionen einer aktuellen Debatte. In: Heinz, W.R.; Kotthoff, H.; Peter, G. (Hg.): Soziale Räume, global players, lokale Ökonomien - Auf dem Weg in die innovative Tätigkeitsgesellschaft? Münster, S. 136-162

Witzel, A., 2000: Das problemzentrierte Interview. In: Forum Qualitative Sozialforschung 1/1 (2000); http:// www.qualitative-research.net/index.php/fqs/article/ view/1132/2519 (download 20.6.13)

Yin, R.K., 2003: Case Study Research. Design and Methods. Thousand Oaks, CA

\section{Kontakt}

Dr. Linda Nierling

Institut für Technikfolgenabschätzung und Systemanalyse (ITAS)

Karlsruhe Institut für Technologie (KIT)

Karlstraße 11, 76133 Karlsruhe

Tel.: +49 721 608-22509

E-Mail: linda.nierling@kit.edu 